\title{
The Importance of FACS Analysis in the Development of Aptamers Specific to Pathogens
}

\author{
Ji-Hea Moon, Giyoung Kim*, Saet Byeol Park, Jongguk Lim, Changyeun Mo \\ National Academy of Agricultural Science, 150 Suin-Ro, Kweonseonku, Suwon, Korea
}

Received: February $25^{\text {th }}$ 2014; Revised: May $19^{\text {th }} 2014$; Accepted: May $27^{\text {th }} 2014$

\section{Abstract}

Purpose: This review aims to introduce aptamers and the methods of its development to improve the sensitivity and selectivity to target bacteria. In this review, we have highlighted current developments and directions in the pathogen detection based on aptamers. Background: Aptamers, the specific nucleic acid sequences, can bind to targets with high affinity and specificity. Some of researches on the use of aptamers for the detection of pathogen have been reported in recent years. Aptamers have more applicability than antibodies for the development of pathogen detection using biosensor; such as easy to synthesis and labeling, lack of immunogenicity, and a low cost of production. However, only few reports on the development and use of aptamers for the detection of pathogen have been published. Review: Aptamers specific to pathogen are obtained by whole-cell systematic evolution of ligands by exponential enrichment (SELEX) process. SELEX process is composed of screening random oligonucleotide bound with target cells, multiple separation and amplification of nucleic acids, final identification of the best sequences. For improving those affinity and selectivity to target bacteria, optimization of multiple separating process to remove unbounded oligonucleotides from aptamer candidates and sorting process by flow cytometry are required.

Keywords: Aptamers, Biosensor, FACS analysis, Pathogen, SELEX

\section{Introduction}

Aptamers are derived from the Latin word "aptus", meaning "to fit" (Stoltenburg, et al., 2007). They are singlestranded oligonucleotides (DNA or RNA) that fold up into 3D structures, allowing them to bind specifically to other target molecules. These aptamers can be isolated from synthetic combinatorial nucleotide libraries by in vitro selection, called to Systematic Evolution of Ligands by Exponential enrichment (SELEX) (Meyer et al., 2013). Aptamers exhibit high-affinity because they are produced by chemical synthesis with extreme accuracy and reproducibility (Stoltenburg, et al., 2007). Since their discovery about twenty years ago, aptamers have been generated against a variety of targets ranging from small molecules, proteins,

\footnotetext{
*Comesponding author: Giyoung Kim

Tel: +82-31-290-1899; Fax: +82-31-290-1900

E-mail: giyoung@korea.kr
}

cancer cells and bacteria (Cibiel et al., 2011). Recently, many reports on the use of aptamers for the detection of pathogenic bacteria have been published (Moon et al., 2011).

Today, many kinds of biomarkers are expressed at the surface of infectious pathogens. There is a high demand for specific ligands against the surface of target bacteria for basic research and monitoring (Cibiel et al., 2011). Antibodies are extensively developed and used as the cell surface biomarkers, but they are difficult to produce with similar specificity and binding affinity, and expensive to produce (Tombelli et al., 2005; Cibiel et al., 2011; Moon et al., 2013). Therefore, aptamers have been described as potent probes, rivaling antibodies. Although development of aptamers to recognize specific membrane proteins of target bacteria is very difficult, their 3D structure leads to a successful binding with pathogen (Meyer et al., 2013). However aptamers, selected against purified domains of bacterial membranes, are sometimes unable to bind with 
the surface of target bacteria. To overcome this drawback, SELEX has been developed to allow aptamer selection possibly to the natural environment (Stoltenburg et al., 2007).

The basic SELEX is composed of repeated five main steps: binding, partition, elution, amplification, and conditioning, but there is no standardized protocol for developing target-specific aptamer. Thus, the optimization of SELEX design and the selection conditions depend on the target itself (Stoltenburg et al., 2007). The whole cell SELEX is that the process whereby live cells are used to select aptamers for target recognition. In this method, nucleic acids bind to molecules on the extracellular surface (Sefah et al., 2010). Therefore, whole cell SELEX is the most optimized in vivo selection for the development of an aptamer specific to pathogenic bacteria. However, the success rate of whole-cell SELEX experiments is about 50 $\%$, because the selection of aptamers against complex structures is very difficult. Therefore, fluorescent-activated cell-sorting (FACS) device was starting to be used to implement this protocol (Mayer et al., 2010). The present review describes the aptamer specific to pathogenic bacteria, whole-cell SELEX, and the FACS analysis for suggesting more effective and successful development of aptamer against pathogen.

\section{Aptamers}

Aptamers are typically below 100 mer, and have the ability to bind to target molecules with dissociation constants $\left(\mathrm{K}_{\mathrm{d}}\right)$ ranging from picomolar to nanomolar (Song et al., 2008). Aptamers are isolated from complex libraries of oligonucleotides (routinely of the order of $10^{15}$ ), generated by combinatorial chemistry, by in vitro process of adsorption, separation, recovery, and re-amplification (James, 2006). Total iterative process is about 7 to 15 rounds. The success of developing an aptamer is driven by the selection conditions: the concentration of bacterial cells and oligonucleotides, incubation time of binding, steps of washing, etc. According to repeat rounds of selection, aptamer candidates actually make three-dimensional shapes, and display a unique combination of double-stranded helical segments, loops, and bulges. Each nucleic acids have hydrogen bonds and electrostatic and van der Waals interactions. This repeated-scaffold folding structure offers a three-dimensional potential for the binding interaction with target molecules. They make very strong affinity and high specificity (Tolumé et al., 2009).

On the basis of these characteristics, aptamers are called to chemical antibodies (Song et al., 2008). However, aptamers have more flexibility in the range of application, a shorter period for production than antibodies. Also, aptamers permit the biochemical manipulation required to various functional groups and specific moieties without any affection to recognize target cells (Sefah et al., 2010). Although aptamers have better binding affinities than those of antibodies, aptamer biosensor could detect only a small range of cells or proteins until now. Because aptamer has been developed recently, antibodies have been studied for a long time. Moreover, research for aptamer has been limited by patents until very recently. Thus antibodies have become well established. In the future, aptamers will be studying and developing worldwide without suppression of patents. For filling in antibody shortcomings, aptamer could be optimized for activity and persistence under real conditions during detecting the target pathogen after discovery (Keefe et al., 2010).

\section{Selection and analytical applications of aptamers for the detection of pathogen}

Since the incidence rate of food poisoning has been increased in the world, pathogen surveillance is the most important to its analysis, control, and prevention (Hamula et al., 2011). Although methods for microbial detection and control are well established, conventional detections are complicated and require multiple steps. Therefore, rapid detection of pathogen has been required and actively developed (Kim et al., 2009, 2013; Moon et al., 2013). Established methods include culture, biochemical tests, and immuno assay based methods. Immuno-biosensor has especially lots of advantages, such as simple, rapid, sensitive, and economical characteristics (Hamula et al., 2011). Biosensor is composed of bioreceptor, transducer, and data recording and display device. In here, bioreceptor is a biological molecular species, such as antibody, enzyme, protein, or nucleic acid for utilizing a biochemical mechanism for recognition (Arora et al., 2013). The antibody has been popularly used to the bioreceptor by the exhibition of high affinity and specific binding capabilities between antigen and antibody. However, the antibody is less effective than aptamer as its own disadvantages: requirements of animal study, and very expensive and laborious production process, a batch-to-batch variation, sensitive to temperature, irreversible denaturation, and limited shelf life (Vo-Dinh 
et al., 2000; Stoltenburg et al., 2007). Also, the binding affinity of aptamers for targets is comparable to or higher than most antibodies. Therefore, aptamers have key advantages over antibodies in their affinity, flexibility, and the nature of in vitro evolution (SELEX) (Hamula et al., 2011).

\section{Whole-cell SELEX}

SELEX is the iterative process for the development of aptamers. Most aptamers selected against bacteria have been evolved using purified target molecules, but their applications to target whole live cells may be limited. Therefore, live bacteria have become increasingly SELEX targets, which allow molecules to be targeted in native conformations on bacterial surface (Hamula et al., 2011). However, the cell surface carries a net negative charge, so that DNA polyanion is bind to cells with difficult, due to charge repulsion (Sefah et al., 2010). Thus, its success rate is about 50\% (Mayer et al., 2010). To solve this problem, researchers has been suggesting a variety kinds of wholecell SELEX process, such as the optimization of PCR amplification, centrifugation for elution of oligonucleotides from cells, and concentration of eluted aptamer candidates (Moon et al., 2013).

The whole cell SELEX process is described in Fig. 1. However, existed whole-cell SELEX limits the selection of sequential aptamer pools. Because aptamer candidates have the binding affinity to the molecule of cell surface, their selectivity could be limited to that of target bacteria or other bacteria, which has similar composition. For the improvement of specificity to targets, counter-SELEX has been developed, in which only unbounded nucleotides to non-target bacteria were selected and amplified for eliminating undesired aptamers. However, complete elimination of variants binding to nonspecific molecules are difficult even though repeated process of SELEX and counter-SELEX (Ohuchi, 2012). Therefore, the post-SELEX modifications are suggested in order to enhance the selectivity and binding affinity. The use of aptamers as probes for flow cytometry has been investigated and now widely being used as the critical process in the whole cell SELEX (Meyer et al., 2013).

\section{Flow cytometry analysis in the whole cell SELEX}

In the whole-cell SELEX, the most critical part is the perfect seperation of bound from unbound or nonspecifically bound oligonucleotides. Flow cytometry has been widely used as the rapid analysis of cell populations on the single cell level. The light scattering and fluorescence signals yield information on various cell characteristics like size, intracellular granularity, and fluorescence tags such as aptamers or proteins (Meyer et al., 2013). Therefore, the flow cytomery isolates the bacteria, bound with fluorescence aptamer, and removes unbound nucleotides. Besides the development of aptamers being specific to pathogens in whole-cell SELEX, flow cytometry assays improved their specificity and affinity to infected samples (Sefah et al., 2010). In the whole-cell SELEX, selection process could be monitored and sorted by flow cytometry where flurescently labeled nucleic acids (aptamer candidates) are incubated, and bound to targets (Meyer et al., 2013). The most important part of the SELEX process is the separation of bound from unbound or unspecifically bound sequences. Thus, this process is the most effective and rapid methods for purifying and identifying bacteriaspecific aptamers (Mayer et al., 2010). Meyer et al. (2013) also described that flow cytometry-based detection of pathogens by aptamers could be possible in real environmental samples. The aptamer, developed by whole cell SELEX with flow cytometry, has more sensitive and specific to target bacteria than antibody, according to its accessibility by chemical synthesis and ease of modifications. These advantages suggested that aptamers present a promising alternative to antibodies in the biosensor system (Moon et al., 2013). Therefore aptamers could compete with antibodies in whole cell SELEX with flow cytometry analysis.

\section{Conclusions}

In summary, this review suggests that aptamers could be an alternative to antibodies by whole-cell SELEX. Using this process, aptamers could be selected because they bind target bacteria with more selective and sensitive than antibodies. However, whole-cell SELEX is carried out under environmental condition that differs from real one. This difference is likely to cause false positive results, such as non-specific bindings of aptamer to non-target bacteria or molecules (Meyer et al., 2013). Aptamer candidates, after the selection in SELEX and counter-SELEX process, might decrease the specificity when used on real samples. This review suggests that flow cytometry analysis could increase the specificity of aptamer candidates, and overcome their limitations. However it requires the optimal combination among SELEX 
process, counter-SELEX process, and flow cytometry analysis. Overall, such improvements to the SELEX should increase the specificity and performance of the final aptamer by allowing for the optimization of both binding affinity and specificity. Aptamers would be a promising group of bioreceptors with their powerful advantages, such as selectivity, sensitivity, stability, the reproducibility of the target binding reaction, and the ease of regeneration of aptamer derivated surfaces. Panels of aptamers targeting higher-order protein complexes, whole cells, bacteria, viruses, parasites or tissues have previously been described (Mayer et al., 2010). According to these results, aptamerbased biosensors have already found broad applications in food and agricultural research. In spite of rapid advances, aptamer-based biosensors are still immature when compared to immunoassays, but new technology (e.g., nanotechnology, or fluorotechnology) would have made an important addition to the analytical and diagnostics (Song et al., 2008).

\section{Conflict of Interest}

The authors have no conflicting financial or other interests.

\section{Acknowledgement}

This work was carried out with the support of "Cooperative Research Program for Agriculture Science \& Technology Development (Project title: Development of a bioprobe for detection of Staphylococcal enterotoxin, Project No: PJ00998701)" Rural Development Administration, Republic of Korea.

\section{References}

Arora, P., Sindhu, A., Kaur, H., Dilbaghi, N and A. Chaudhury. 2013. An overview of transducers as platform for the rapid detection of foodborne pathogens. Applied Microbiology and Biotechnology 97:1829-40.

Cibiel, A., Dupont, D. M and F. Ducongé. 2011. Methods to identify aptamers against cell surface biomarker. Pharmaceuticals 4:1216-35.

Hamula, C. L. A., Zhang, H., Li, F., Wang, Z., Le, X. C and X. F. Li. 2011. Selection and analytical applications of aptamers binding microbial pathogens. Trends in Analytical Chemistry 30(10):1587-97.
James, W. 2006. Aptamers. In:Encyclopedia of Analytical Chemistry, ed. R.A. Meyers, pp. 1-23. Oxford, UK: John Willey \& Sons Ltd.

Keefe, A. D., Pai, S and A. Ellington. 2010. Aptamers as therapeutics. Nature Reviews Drug Discovery 9:537-550.

Kim, G., Moon, J. H., Hahm, B. K., Morgan, M., Bhunia, A and A. S. Om. 2009. Rapid detection of Salmonella Enteritidis in pork samples with impedimetric biosensor: effect of electrode spacing on sensitivity. Food Science and Biotechnology 18(1):89-94.

Kim, G., Yang, G., Park, S. B., Kim, Y. H., Lee, K. J., Son, J. Y., Kim, H. J and S. R. Lee. 2011. Rapid detection kit for Salmonella Typhimurium. Journal of Biosystems Engineering 34:140-6.

Mayer, G., Ahmed, M. S. L., Dolf, A., Endl, E., Knolle, P. A and M. Famulok. 2010. Fluorescence-activated cell sorting for aptamer SELEX with cell mixtures. Nature Protocols 5(12):1993-2004.

Meyer, M., Scheper, T and J. G. Walter. 2013. Aptamers: versatile probes for flow cytometry. Applied Microbiology and Biotechnology 97:7097-109.

Moon, J., Kim, G., Lee, S and S. Park. 2013. Identification of Salmonella Typhimurium-specific DNA aptamers developed using whole-cell SELEX and FACS analysis. Journal of Microbiological Methods 95:162-6.

Ohuchi, S. 2012. Cell-SELEX Technology. Bioresearch 1(6):265-72.

Sefah, K., Shangguan, D., Xiong, X., O'Donoghue, M. B and W. Tan. 2010. Development of DNA aptamers using cell-SELEX. Nature Protocols 5(6):1169-85.

Song S., Wang, L., Li, J., Zhao, J and C. Fan. 2008. Aptamerbased biosensors. Trends in Analytical Chemistry 27(2):108-117.

Stoltenburg, R., Reinemann, C and B. Strehlitz. 2007. SELEX-A (r)evolutionary method to generate highaffinity nucleic acid ligands. Biomolecular Engineering 24:381-403.

Tombelli, S., Minunni, M and M. Mascini. 2005. Piezoelectric biosensors: strategies for coupling nucleic acids to piezoelectric devices. Methods 37:48-56.

Toulmé, J. J., Daguer, J. P and E. Dausse. 2009. Aptamers: Ligands for all reasons. In: Aptamers in Bioanalysis, ed. M. Mascini. P.p. 3-30. New Jersey, NJ: John Wiley \& Sons, Inc.

Vo-Dinh, T and B. Cullum. 2000. Biosensors and biochips: advances in biological and medical diagnostics. Fresenius' Journal of Analytical Chemistry 366:540-51. 